\title{
Chemically induced grain boundary dynamics, forced motion by curvature, and the appearance of double seams
}

\author{
PAUL C. FIFE ${ }^{1}$ and XIAO-PING WANG \\ ${ }^{1}$ Mathematics Department, 155 South 1400 East, University of Utah, \\ Salt Lake City, UT 84112-0090, USA \\ ${ }^{2}$ Mathematics Department, The Hong Kong University of Science and Technology, \\ Clear Water Bay, Kowloon, Hong Kong
}

(Received 29 September 2000; revised 24 August 2001)

\begin{abstract}
The free boundary model of diffusion-induced grain boundary motion derived in Cahn et al. [3], Fife et al. [6] and Cahn \& Penrose [4] is extended, in the case of thin metallic films, to account for bidirectional motion, together with the appearance of S-shapes and double seam configurations. These are often observed in the laboratory. Computer simulations based on the extended model are given to illustrate these and other features of bidirectional motion. More generally, the extension accounts for the motion of grain boundaries whose traces on the film's surface are curved. The new free boundary model is one of forced motion by curvature, the forcing term possibly changing sign due to the bidirectionality. The thin film model is derived systematically under explicit assumptions, and an adjustment for grooving is included.
\end{abstract}

\section{Introduction}

Boundaries between grains (separate crystals) in a metallic alloy specimen may migrate due a variety of reasons. In particular the following phenomenon, called Diffusion Induced Grain Boundary Motion (DIGM), is well known and has been studied extensively, both experimentally and theoretically. Atoms of a solute species (one of the species making up the alloy) in a vapour which is in contact with the metallic surface may enter the grain boundaries and diffuse rapidly along them. The resulting presence of solute atoms may, by inducing stresses in the material adjacent to (or in) the boundaries, cause the latter to migrate, eating away one crystalline grain while building up the next. See the diagram in Figure 1.

A quantitative model of DIGM was developed in Cahn et al. [3]. It allows the velocity of this migration to be predicted on the basis of known physical parameters and the local concentration of solute. The grain boundary itself is considered to be of finite thickness, and since it involves a continuously varying order parameter measuring crystallinity, the model is of the 'phase-field' type. It consists of nonlinear partial differential equations for (1) the concentration $u$ of solute, and (2) the order parameter, both being functions of position in the grain boundary and time. 
This phase-field model was further developed in Fife et al. [6], especially in the context of curved boundaries in a 2D geometry, and then through a systematic approximation procedure reduced to a free boundary model. In this latter version, the grain boundary has zero thickness. It is represented by a 'free boundary' curve. The law of motion of this free boundary specifies that its normal velocity is the sum of two terms: one of them proportional to the mean curvature, and the other dependent on the concentration of solute at that point. In addition, the solute diffuses along the boundary and its concentration is reduced due to its incorporation into the growing daughter grain.

We thus obtained [6] a pair of equations for the evolution of the free boundary and the concentration $u$. In dimensionless form it is:

$$
\begin{aligned}
& \rho v=\kappa+u^{2}, \\
& u_{s s}-v u=0 .
\end{aligned}
$$

Here $v$ is the normal velocity of the curve, $\kappa$ is its curvature, $\rho$ is a dimensionless material constant, and $s$ measures arclength along the curve.

The contact curve where the grain boundary in the metallic specimen meets the specimen's face adjacent to the vapour reservoir (seen on the top face in Figure 1) is in general not really a curve, but rather a traveling groove carved into the metal by the action of surface diffusion (see Figure 3 in $\S 5$ ). The phenomenon of grooving was first given a mathematical basis by Mullins $[9,10]$. The existence of grooves is due to the diffusion of material on the surface of a crystalline grain. That diffusion tends to smooth out sharp corners, such as corners which would be evident where the grain boundary meets the specimen face were there no grooves. More discussion is given in $\S 5$. This grooving effect was disregarded in Cahn et al. [3] and Fife et al. [6], the contact being represented as a curve, but was studied carefully in Cahn \& Penrose [4]. This latter paper builds on the model given in Fife et al. [6].

Beyond this, there are two important limitations to the theory developed in Fife et al. [6] and Cahn \& Penrose [4]. The first one is that grain boundaries are only considered whose contact curves (or grooves) on the face of the metal are straight (in Figure 1 it is curved), and the second one is that only unidirectional motions of the grain boundary were allowed. Both of these limitations are overcome in this paper.

Consider the first limitation. In addition to the assumption of straightness, it was supposed in Fife et al. [6] and Cahn \& Penrose [4] that at each point on the grain boundary, its motion is in a direction perpendicular to the contact line (or groove). A rigorous existence theory, in fact, was given for configurations of this type which are steady, in that they move in a direction parallel to the surface without changing shape or speed.

This limitation is a geometical one; it reduces the problem to the consideration of an evolving curve in a plane, namely the curve formed by the intersection of the grain boundary with a plane perpendicular to the contact line (hence also to the metal surface). As far as the derivation of the free boundary model from the phase-field model given in Fife et al. [6] is concerned, this restriction is easily surmounted (§2). Each step in the derivation generalizes to the case when the phase-field version of the grain boundary is a thin layer of any shape within a three-dimensional metal plate. The reduction is made from 


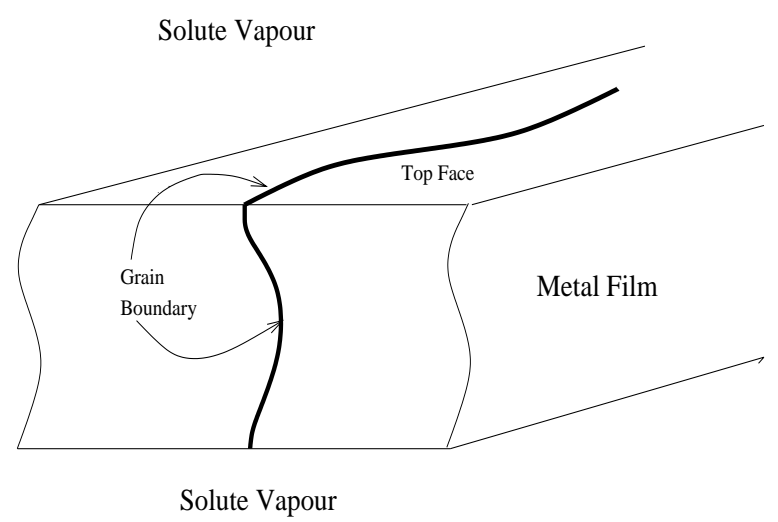

FIGURE 1. Grain boundary cutting through a section of metallic film.

this layer to a free boundary which is a mobile surface imbedded in three-dimensional space. The equations of motion of this free boundary are the same as before, except that the curvature term is now the sum of the two principal curvatures of this surface, and the diffusion term is the analogous diffusion of the solute within the surface.

In the investigation of the steady configurations mentioned above, both papers [6, 4] consider two cases: (i) the case when the specimen is a film so thin that the grain boundary can extend from one face all the way to the other (as in Figure 1); and (ii) relatively thick films or plates, for which the migrating boundary cannot extend all the way through; instead it trails behind the moving contact point, eventually curving to become parallel to the face of the specimen. The first case was called the 'connecting' case, the second one the 'trailing' case. When the film is thin enough and the grooving angle is zero, the grain boundary's intersections with planes perpendicular to the faces are expected to be approximately straight line segments connecting the two faces, at least after initial transients have relaxed. This was the case assumed in the original model [3] (in fact there was no curvature to the boundary at all). The justification of this claim is given in $\S 3$ below, on the basis of explicit assumptions designed to eliminate transient behaviour.

In this paper, we adopt this thin film assumption, but contrary to the previous papers, now allow the grain boundary to be curved in the plane of the specimen's faces. Thus the important shape dynamics is similar to that of the curve on the top face in Figure 1, namely that seen by an observer away from the specimen, looking at its surface. This provides a perspective of the grain boundary and its motion which is different from that in Cahn et al. [3], Fife et al. [6] and Cahn \& Penrose [4]. Rather than considering the boundary's motion within the material specimen, we develop the law of motion and properties of the evolving trace of the grain boundary on the face of the specimen. This new perspective is what is often seen in experiments.

Our free boundary problem, derived formally on the basis of the thin film assumption and an assumption related to the disappearance of transients, says that the trace of the grain boundary on the surface of the specimen obeys a forced motion by curvature law. It is derived first for the case of zero grooving angle, and later $(\S 5)$ conditions are given under which a similar free boundary problem applies with the angle nonzero but small. 
Both of these derivations, when the angle is zero and then nonzero, relate to what we envisage as the motion after rapid transients have disappeared. In the zero angle case, that implies, among other things, that the grain boundary inside the film is approximately perpendicular to the face. But geometrical conditions such as this are not assumed. They are rather derived in turn from a more basic assumption (see §3). To explain this, we consider a family of solutions of the 3D free boundary problem in a film, the parameter of the family being the film thickness $h$. Then we assume that that each term in the equations of motion is bounded independently of $h$. For example, we assume that the curvature of the grain boundary is bounded independently of $h$. Initial grain boundary configurations concocted so that their curvatures violate this assumption can be imagined, but it is expected that during the associated subsequent motion, transient effects will cause these high curvatures to relax.

In the case when the the groove angle is not zero, there are complications due to the fact that it may happen that the two groove roots (the ones on the upper and lower faces; see Figure 3) may be close to each other. When that happens, a large curvature is automatically induced on the grain boundary between the two roots. This in turn prohibits any motion unless the forcing due to solute concentration is large enough [4]. Our free boundary problem is therefore only valid if this condition is fulfilled. We must combine this threshold condition with a non-transient assumption analogous to that described above. This is done in $\S 5.4$ by means of a 'reference profile', an explicit configuration inside the film which is a solution of the $3 \mathrm{D}$ problem when the trace on the film surface is straight. A second complication which arises in this case is that the depths of the grooves may change in time. A discussion of this, and an argument as to why this effect is typically small, are given in $\S 5$.

The second major limitation to the theory in Fife et al. [6] is that only unidirectional motions of the grain boundary were allowed. In fact, at the very outset, the partial differential equations developed in Cahn et al. [3] were used together with boundary conditions and interaction terms which were appropriate for motion in a specific direction relative to the grain boundary. If the motion were in the opposite direction, those terms would have to be changed.

One of the aims of this paper is to eliminate this hypothesis, allowing for movements in either direction. See the discussion in $\S 4$. Configurations with variable direction of migration are commonplace in experiments $[2,5,7,8,11]$.

We provide computer simulations of solutions of our Free Boundary Problem (FBP) for various prototypical grain boundary configurations. These are suggestive of common observations such as $S$-shaped boundaries and the formation of double seams by one portion of the grain boundary doubling back to abut a region which has already been covered by another portion. These simulations posit two or more adjacent intervals of the initially straight grain boundary, each spawning migration in its own direction, that direction differing from one interval to its neighbor.

In general, the transition point between two adjacent portions of the mobile boundary, marking the point at which the direction of motion changes, will itself migrate; this is exemplified by the simulations in $\S 6.3$. However, symmetry of the movement resulting from imposed symmetric initial conditions may serve to pin this transition point, as seen in Figures 4-8. 
Here is an outline of the paper. To incorporate our new perspective into the analysis, we present in $\S 2$ the three-dimensional analogue of the FBP which was derived in Fife et al. [6]. That derivation was exceedingly complex, due largely to the degeneration of the assumed mobility of the solute atoms as they enter the crystalline grain from the grain boundary. Therefore, rather than repeating the derivation in the $3 \mathrm{D}$ context, we shall simply indicate the specific changes to be made in Fife et al. [6] to adapt that argument to the present scenario. Most of the details are put into an appendix.

We then proceed in $\S 3$ to carry through an approximation procedure valid when the material is a thin film. This analysis reduces the dimension from 3 back to 2. At this stage we are continuing to neglect the grooving effect, which is taken up in $\S 5$. The resulting FBP so derived governs the evolution of the trace of the free boundary on the film's surface.

Actual physical processes involving chemically induced grain boundary motion (DIGM) are subject to at least two further considerations beyond the mere statement of the FBP derived in $\S 3$ : the fact that they often move in opposite directions in different patches or segments of the grain boundary, and the fact that the law of motion changes once a grain boundary impinges on a part of the material which has already been covered by the DIGM process. These extra considerations are discussed in $\S 4$ in the context of our model; in particular, the appropriate modifications to the FBP are outlined.

In $\S 5$, the grooving phenomenon is discussed in our same context, and a physical scenario is suggested under the FBP which was derived in $\S 3$ remains valid when grooving is present. Observed effects of grooving on the motion of grain boundaries have been brought out in Cahn \& Penrose [4]. For example, the analysis in that paper reflects the observation that the film must attain a certain minimal thickness before motion is possible. Accordingly, in $\S 5$ thicknesses larger than the critical one are considered.

The simulations are presented in $\S 6$. They involve three prototypical features, as mentioned above. A summary is given in the discussion, $\S 7$.

\section{The free boundary problem in three dimensions}

In Fife et al. [6], a free boundary problem was derived to describe the diffusion-induced motion of grain boundaries. The derivation consisted of a formal asymptotic reduction of a phase field model which had been introduced in Cahn et al. [3]. The result of the reduction is (1.1), (1.2).

The phase field model is a system of two nonlinear partial differential equations of parabolic type for the concentration of the solute species and an order parameter representing the crystallinity of the material. The grain boundary, at this stage, was considered to have finite thickness, and these two functions (concentration and order parameter) were defined in the region representing the grain boundary. The magnitude of the order parameter is least in the interior of the grain boundary, and maximal where the material becomes part of the abutting grains. The partial differential equation (PDE) for the order parameter is an Allen-Cahn (Ginzburg-Landau) equation with forcing term dependent on the concentration. Therefore, it is no surprise to one familiar with internal layers that its formal reduction involves curvature-dependent motion of the free boundary.

The PDE for the concentration is an equation which reflects the diffusion of solute 
atoms, with diffusivity vanishing when the atoms enter the crytalline grain. In addition, there is another term coupling the concentration field to the order parameter field.

This was a $2 \mathrm{D}$ theory, all the fields being assumed constant in the third direction, parallel to the faces.

The asymptotic reduction in Fife et al. [6] consisted of an extremely involved singular limit as the thickness of the grain boundary and another parameter measuring the elastic effects tended to zero.

The analogous 3D theory in thin films, as we shall show, takes the form of the following free boundary problem for the motion of the surface $\Gamma(t)$, which represents the grain boundary, and the concentration $u$ of solute in the grain boundary:

$$
\begin{gathered}
\rho v=\kappa_{1}+\kappa_{2}+f(u), \\
\Delta_{\Gamma} u-v u=0 .
\end{gathered}
$$

Here $v$ is the normal velocity of $\Gamma, \Delta_{\Gamma}$ is the Laplace operator on the surface $\Gamma(t)$, and $\kappa_{i}$ are curvatures of $\Gamma(t)$ in any two orthogonal directions (in the surface). The parameter $\rho$ is a dimensionless combination of material constants defined in Fife et al. [6]. Although there are no explicit time derivatives here, this is a dynamical problem because of the presence of $v$. In fact, (2.1) tells us the normal velocity of $\Gamma$ as a function of the mean curvature of $\Gamma$ and the local concentration of solute. In turn, (2.2) expresses rapid diffusion of the solute along $\Gamma$, together with its loss, during the motion, due to the solute being trapped in the growing grain. This latter effect is expressed by the term $v u$.

In addition, there are boundary conditions on the film's faces. The grain boundary must meet them at a prescribed angle of grooving $\theta_{g}$ at the point where it intersects them, and $u=1$ on the top face. If the bottom face is also in contact with a solute reservoir, then $u=1$ there as well. If it is in contact with an inert material, then the normal derivative of $u$ vanishes there. In Fife et al. [6] and Cahn \& Penrose [4], $f(u)$ was taken to be $u^{2}$ in accord with the theory in Cahn et al. [3], but here it could be any nonnegative increasing function.

The 2D free boundary problem (1.1), (1.2) derived in Fife et al. [6] is like this, except that $\Gamma(t)$ is a mobile curve, hence only one curvature appears in (2.1), and the Laplacian in (2.2) is replaced by the second derivative with respect to arclength.

In Appendix A, it is shown how the derivation in Fife et al. [6] can be modified to fit the present $3 \mathrm{D}$ scenario, resulting in (2.1), (2.2).

\section{The thin film approximation when $\theta_{g}=0$}

As mentioned before, we suppose the specimen $\Omega$ to be a film occupying the set in the space $\left(x_{1}, x_{2}, y\right)$ (dimensionless variables) given by $\{-2 h<y \leqslant 0\}$ (Figure 2). We now assume $h \ll 1$. The free boundary $\Gamma(t)$ extends through $\Omega$, so has traces on the top and bottom faces $\{y=0\}$ and $\{y=-2 h\}$. In this section, we restrict $\theta_{g}=0$ and disregard the depth of any groove; other cases are considered in $\S 5$.

We shall give a formal argument in this section, based on (1) the smallness of $h$ and (2) assumptions designed to eliminate transient behaviour, that the evolution of the surface $\Gamma$ governed by (2.1), (2.2) may in normal circumstances be reduced to a simpler evolution of the curve $\Gamma_{0}(t)$, which is the intersection of $\Gamma$ with the upper surface $\{y=0\}$ of 


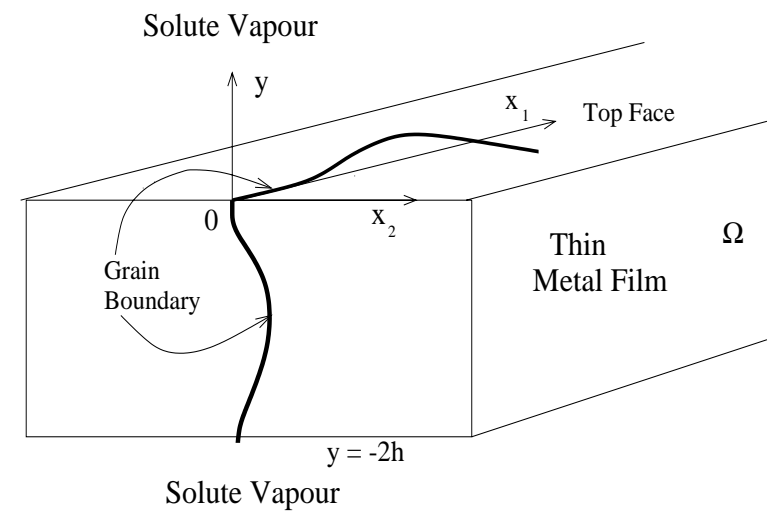

Figure 2. Coordinate system used in the thin film.

the material. Thus the dynamical behaviour of the part of the surface $\Gamma$ lying below that upper plane will have little effect on the motion of $\Gamma_{0}(t)$, which is approximately autonomous. That part will be approximately generated by lines perpendicular to the surface. Our argument is summarized below in $\S 3.7$.

Regarding the exceptional circumstances, one can imagine a sequence of values of $h$ tending to 0 , together with a sequence of initial conditions to be contrived, for example, so that although $\Gamma_{0}(0)$ may be a fixed curve, $\kappa_{1}+\kappa_{2}$ becomes arbitrarily large on the sequence, and $\Gamma$ is very far from being normal to the upper surface. However, our expectation is that any such initial abnormalities will quickly be damped out by transient behaviour. We consider only motions for which such transients are no longer present. We will spell out below an assumption on the solution designed to eliminate them. Roughly speaking, it is that the terms in the evolution equations (2.1), (2.2) are smooth functions with regularity properties independent of $h$.

First, we rewrite (2.1) in the form of differential operators valid locally, using the variables $\left(x_{1}, x_{2}, y\right)$. Let $H$ be the mean curvature of $\Gamma$, so that $\kappa_{1}+\kappa_{2}=2 H$, and we make that replacement in (2.1). With no loss of generality, we assume that the origin $(0,0,0)$ lies on $\Gamma_{0}(0)$, and that $\Gamma_{0}(0)$ is tangent to the $x_{1}$-axis at the origin. Then for small $\left(x_{1}, y\right)$ we may represent $\Gamma(0)$ as

$x_{2}=X_{2}\left(x_{1}, y\right), \quad$ where $\quad X_{2}(0,0)=0, \quad \partial_{x_{1}} X_{2}(0,0)=0, \quad \partial_{y} X_{2}\left(x_{1}, 0\right)=\partial_{y} X_{2}\left(x_{1},-2 h\right)=0$.

The last normality requirement in (3.1) was mentioned following (2.2) and is the same as equation (2.76) of Fife et al. [6].

The mean curvature $H$ of $\Gamma(0)$ can be expressed as

$$
H\left(x_{1}, y\right)=\left(a_{11} \partial_{x_{1}}^{2}+a_{12} \partial_{x_{1}} \partial_{y}+a_{22} \partial_{y}^{2}\right) X_{2}\left(x_{1}, y\right),
$$

where the coefficients $a_{i j}$ are nonlinear functions of $\nabla X_{2}\left(x_{1}, y\right)$. For example,

$$
a_{12}=-\frac{2\left(\partial_{x_{1}} X_{2}\right)\left(\partial_{y} X_{2}\right)}{\left(1+\left(\partial_{x_{1}} X_{2}\right)^{2}+\left(\partial_{y} X_{2}\right)^{2}\right)^{3 / 2}}
$$

The other coefficients $a_{11}$ and $a_{22}$ are positive and bounded below as well as above. 
Thus (2.1) becomes

$$
\rho v=2\left(a_{11} \partial_{x_{1}}^{2}+a_{12} \partial_{x_{1}} \partial_{y}+a_{22} \partial_{y}^{2}\right) X_{2}\left(x_{1}, y\right)+f(u) .
$$

\subsection{Basic assumption}

All terms in (3.4) and (2.2), together with their first spatial derivatives, are bounded independently of $h$.

We draw the following consequences.

\section{$3.2 \Gamma$ is almost vertical}

By (3.1), $\partial_{y} X_{2}=0$ at $y=0$ and $-2 h$, so by the mean value theorem, for each $x_{1}$ there is a value of $y$ at which $\partial_{y}^{2} X_{2}=0$. By the basic assumption and the boundedness from below of $a_{22}$, we know that $\partial_{y}^{3} X_{2}$ is bounded. We therefore have the estimate

$$
\left|\partial_{y}^{2} X_{2}\right| \leqslant C h
$$

for all points. Integrating from $y=0$ and using (3.1), we obtain

$$
\left|\partial_{y} X_{2}\left(x_{1}, y\right)\right| \leqslant C h^{2},
$$

and one more time,

$$
\left|X_{2}\left(x_{1}, y\right)-X_{2}\left(x_{1}, 0\right)\right| \leqslant C h^{3} .
$$

Since the choice of origin was arbitrary, similar estimates hold everywhere on $\Gamma(t)$, and for all $t$.

These order relations (3.5)-(3.7) may be differentiated with respect to $x_{1}$ or $t$.

\subsection{The curvature of $\Gamma$ is close to that of $\Gamma_{0}$}

Similarly, we have from (3.3) and (3.6) (differentiated) that $\left|a_{12} \partial_{x_{1}} \partial_{y} X_{2}\right| \leqslant C h^{2}$; combining this with (3.5), we have

$$
\left|H\left(x_{1}, y\right)-H_{0}\left(x_{1}\right)\right| \leqslant C h,
$$

where

$$
H_{0}\left(x_{1}\right)=a_{11} \partial_{x_{1}}^{2} X_{2}\left(x_{1}, 0\right) \equiv \kappa_{0}\left(x_{1}\right),
$$

and $a_{11}$ is to be evaluated with $\partial_{y} X_{2}=0$. This is the curvature of $\Gamma_{0}$.

\subsection{The velocity of $\Gamma$ is close to that of $\Gamma_{0}$}

Let $s_{0}$ be an arclength parameter for $\Gamma_{0}$. We may represent points on $\Gamma(t)$ by $\left(s_{0}, t, y\right)$.

We now derive a relation between the normal velocity vector $\boldsymbol{v}\left(s_{0}, t, y\right)$ of $\Gamma(t)$ and the normal velocity vector $\boldsymbol{v}_{0}\left(s_{0}, t\right)$ of $\Gamma_{0}(t)$.

Let $\boldsymbol{X}_{0}\left(s_{0}, t\right)$ be the 3D position vector corresponding to the point $\left(s_{0}, t\right)$. Thus

$$
\boldsymbol{X}_{0}\left(s_{0}, t\right) \in \Gamma_{0}(t) \text { for all }\left(s_{0}, t\right) .
$$


Let $\Gamma_{1}\left(s_{0}, t\right)$ be the intersection of $\Gamma(t)$ with the plane $P\left(t, s_{0}\right)$ perpendicular to $\Gamma_{0}(t)$ at that point. Points on $\Gamma_{1}$ may be denoted by

$$
\boldsymbol{X}\left(s_{0}, t, y\right)=\boldsymbol{X}_{0}\left(s_{0}, t\right)+y \boldsymbol{e}_{y}+\boldsymbol{\Xi}\left(s_{0}, t, y\right), \quad-2 h \leqslant y \leqslant 0,
$$

where $\boldsymbol{e}_{\boldsymbol{y}}$ is the unit vector in the $y$-direction, and $\boldsymbol{\Xi} \perp \boldsymbol{e}_{y}$. Here the vector $\boldsymbol{\Xi}$ is the (horizontal) deviation of the point on $\Gamma(t)$ at depth $y$ from the projection, to depth $y$, of its associated point $\boldsymbol{X}_{0}$ on the top surface.

By (3.6) and (3.7) we may write $\boldsymbol{\Xi}=h^{2} \xi$, where $|\xi| \leqslant C h$ and $\left|\partial_{y} \xi\right| \leqslant C$.

The normal velocity vector can be represented as

$$
\boldsymbol{v}\left(s_{0}, t, y\right)=\left(\partial_{t}+\alpha\left(s_{0}, t, y\right) \partial_{s_{0}}+\beta\left(s_{0}, t, y\right) \partial_{y}\right) \boldsymbol{X}\left(s_{0}, t, y\right),
$$

where $\alpha$ and $\beta$ are chosen so that $\boldsymbol{v}$ is normal to the surface $\Gamma$. The condition that $\boldsymbol{v}$ be normal to $\Gamma$ is

$$
\boldsymbol{v} \cdot \partial_{s_{0}} \boldsymbol{X}=\boldsymbol{v} \cdot \partial_{y} \boldsymbol{X}=0
$$

since $\partial_{s_{0}} X$ and $\partial_{y} X$ are tangential to $\Gamma$. These two equations (3.13), with $v$ given by (3.12), can be solved for $\alpha$ and $\beta$. In the expression (3.12), we make the substitution (3.11) to obtain

$$
\boldsymbol{v}\left(s_{0}, t, y\right)=\left(\partial_{t}+\alpha \partial_{s}\right) \boldsymbol{X}_{0}\left(s_{0}, t\right)+\beta \boldsymbol{e}_{y}+O\left(h^{2}\right) .
$$

The second of equations (3.13) now yields $\beta=O\left(h^{2}\right)$ and the first yields

$$
\boldsymbol{v} \cdot \partial_{s_{0}} \boldsymbol{X}_{0}=O\left(h^{2}\right) \text {. }
$$

The analogs of (3.12), (3.13) for the velocity of $\Gamma_{0}$ are

$$
\begin{gathered}
\boldsymbol{v}_{\mathbf{0}}\left(s_{0}, t\right)=\left(\partial_{t}+\alpha_{0}\left(s_{0}, t\right) \partial_{s_{0}}\right) \boldsymbol{X}_{\mathbf{0}}\left(s_{0}, t\right), \\
\boldsymbol{v}_{\mathbf{0}}\left(s_{0}, t\right) \cdot \partial_{s_{0}} \boldsymbol{X}_{\mathbf{0}}\left(s_{0}, t\right)=0 .
\end{gathered}
$$

From (3.14) we have

$$
\boldsymbol{v}\left(s_{0}, t, y\right)=\left(\partial_{t}+\alpha\left(s_{0}, t, y\right) \partial_{s_{0}}\right) \boldsymbol{X}_{\mathbf{0}}\left(s_{0}, t\right)+O\left(h^{2}\right) .
$$

Subtract (3.16) from (3.18) and (3.17) from (3.15) to obtain

$$
\begin{gathered}
\boldsymbol{v}\left(s_{0}, t, y\right)-\boldsymbol{v}_{0}\left(s_{0}, t\right)=\left(\alpha-\alpha_{0}\right) \partial_{s_{0}} \boldsymbol{X}_{0}\left(s_{0}, t\right)+O\left(h^{2}\right), \\
\left(\boldsymbol{v}-\boldsymbol{v}_{0}\right) \cdot \partial_{s_{0}} \boldsymbol{X}_{0}=O\left(h^{2}\right) .
\end{gathered}
$$

Since $\partial_{s_{0}} \boldsymbol{X}_{0}$ is a unit vector, we may take the scalar product of (3.19) with $\partial_{s_{0}} \boldsymbol{X}_{0}$ to obtain $\left|\alpha-\alpha_{0}\right| \leqslant C h^{2}$, hence

$$
\boldsymbol{v}=\boldsymbol{v}_{0}+O\left(h^{2}\right)
$$

\section{$3.5 u$ is close to 1}

Let us now consider the distribution $u\left(s_{0}, t, y\right)$ of solute along the the grain boundary as a function of $y$, for fixed $\left(s_{0}, t\right)$. By our main assumption, we only consider solutions such that all terms in $(2.2)$ are $\leqslant O(1)$; in particular, $u_{y y} \leqslant O(1)$. At the upper face $\{y=0\}$ 
we have $u=1$ and at the lower face either $u=1$ (if the vapour is in contact with the specimen there) or $u_{y}=0$ (if it is in contact with a neutral material). In either case there is a location where $u_{y}=0$, so that since the film has thickness $h,\left|u_{y}\right| \leqslant O(h)$. Integrating again, we conclude that

$$
|u-1| \leqslant O\left(h^{2}\right) .
$$

Thus in (2.1), $f(u)=f(1)+O\left(h^{2}\right)$.

\subsection{The evolution law for $\Gamma_{0}$}

All in all, from (3.22), (3.21), (3.8) and (3.9), we find that (2.1) becomes $\rho v_{0}=\kappa_{0}+f(1)+$ $O(h)$, where $v_{0}$ is the normal velocity of $\Gamma_{0}, v_{0}=\boldsymbol{v}_{0} \cdot \boldsymbol{v}_{0}, \boldsymbol{v}_{0}$ being the unit normal. We take $f(1)=1$, so that $\rho v_{0}=\kappa_{0}+1+O(h)$. We now rescale the time variable to eliminate the factor $\rho$. Thus our approximate free boundary problem is

$$
v_{0}=\kappa_{0}+1 .
$$

The second equation (2.2) is no longer applicable. It represents the conservation of solute species at an interior point of the grain boundary. But at the top face, solute really is not conserved; there is an infinite reservoir of solute atoms at concentration unity which replenishes any lost through the migration of $\Gamma_{0}$.

\subsection{Summary}

The starting point in this derivation was the 3D FBP (2.1), (2.2). We introduced the coordinate system shown in Figure 2 and wrote the equation (3.4) for the velocity of the grain boundary in terms of that system. We envisaged a family of configurations in which the thickness $2 h$ of the film tended to zero. Thus in fact the solution depends on $h$. Our main assumption, designed to eliminate transients, was that the terms in the law of motion (3.4), (2.2) are all bounded independently of $h$. Using this assumption, we derived estimates, in terms of $h$, on the deviation from verticality of $\Gamma$ in the film, the deviation of the curvature of $\Gamma$ from that of $\Gamma_{0}$, the same for the normal velocities, and the deviation of $u$ from 1 . These estimates yielded an evolution law for $\Gamma_{0}$ which contained error terms which vanish as $h \rightarrow 0$. Neglecting them produced our final model (3.23).

\section{Changes of direction and obstacles}

When the DIGM process initially causes a grain boundary to move, the direction of motion could depend on microscopic asymmetries which are not accounted for in our theory. Along a given grain boundary, the direction is commonly observed to change from one location to another. Thus one segment of the boundary may be seen to move to the right (say), while an adjacent patch moves to the left. We must decide on modifications to our free boundary problem (3.23) to account for bidirectional motion.

We conceive of the trace of the grain boundary $\Gamma_{0}(t)=\Gamma^{+}(t) \cup \Gamma^{-}(t)$ as being composed of two parts, $\Gamma^{+}(t)$ being the part which moves one way (we shall say in the "positive direction') and $\Gamma^{-}(t)$ moving in the other direction. On $\Gamma^{+}(t)$, (3.23) models the motion. 
For convenience we drop the subscript ' 0 ' to obtain

$$
v=\kappa+1 \text { on } \Gamma^{+}(t)
$$

Here $v>0$; it is normal velocity in the positive direction, and the convention is that $\kappa>0$ if the centre of curvature is on the positive side of $\Gamma^{+}$.

On $\Gamma^{-}$, however, we shall still retain the same notion of positive direction and the same curvature sign convention, so that $v<0$. The normal velocity (considered positive) is now $-v$ and the curvature relative to the new direction is now $-\kappa$. We therefore obtain $-v=-\kappa+1$, or

$$
v=\kappa-1 \text { on } \Gamma^{-}(t)
$$

Near transition points where the direction changes, we expect the velocity to be small. However, the original derivation in [3] on the basis of this mechanism resulted in no dependence of the forcing term on magnitude of the velocity. Therefore, we shall take the forcing term to change discontinuously from +1 to -1 as one passes through such a point. At the transition point itself, the effect of curvature will still be felt, however. This prevents corners from occurring there; we require that $\Gamma_{0}$ be everywhere continuously differentiable.

The transition point itself may move, and an example where this occurs is investigated in $\S 6.3$.

The part of the material which has been swept over by a migrating grain boundary during DIGM is closer to equilibrium, and a new DIGM-produced grain boundary coming to the edge of this region would operate by a different law of motion. If the material is in equilibrium relative to the solute concentration in the adjacent vapour, then the grain boundary would have no DIGM forcing, and would be subject to motion by curvature only; the last term in (4.1) or (4.2) would be absent.

In typical cases, this means that a new grain boundary approaching such an equilibrium region would be prevented from entering it. This obstacle phenomenon is relevant to double seam formation. We incorporate it in the simulations of $\S 6.1$.

\section{Adjustment for grooving}

\subsection{Background}

It is generally the case that the intersection of a grain boundary with the surface of the metal specimen is a V-shaped groove extending into the specimen some distance $d$ (see Figure 3). The centre line of the groove may deviate from the normal to the surface by some typically small angle $\theta_{g}$.

Mullins $[9,10]$ gave a theory of the dynamics of such grooves, based on the phenomenon of surface diffusion. The importance of the grooving effect depends on the material and the temperature. Mullins [10] found a connection between the depth $d$ and the velocity of the grain boundary when the latter moves steadily as a whole in a direction parallel to the surface. He also considered [9] a groove which is stationary in this sense, but whose depth increases in time. Then $d \sim t^{1 / 4}$. Because of the small exponent, Mullins argued that at times of practical interest, the rate of increase of $d$ is so small that it can be considered stationary. In fact, he provided a rough estimate of that stationary value. By the same 


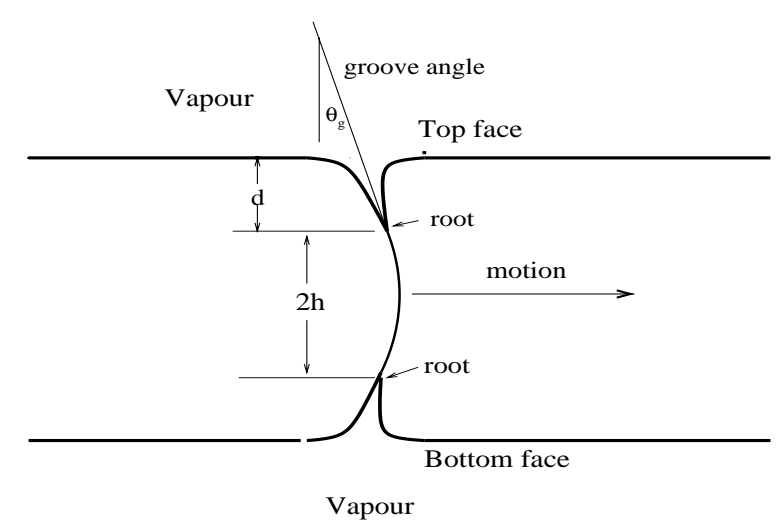

FIGURE 3. Film section showing the grooving phenomenon.

token, when $t$ is small $d$ increases rapidly at a rate $\sim t^{-3 / 4}$. In other words, the groove depth $d$ relaxes rapidly to its preferred value.

Mullins also [10] that the groove angle $\theta_{g}$, for steady motions, is approximately independent of the velocity, a typical value being around .05 radians.

In our development, we assume that $d$ is constant. If, for example, a migration is initiated by immersing a sample with existing grain boundary in a solute reservoir, we assume that the groove depth has already reached its characteristic depth before the immersion time. The subsequent motion of the boundary will affect $d$, but not instantaneously; there will be a time lag. The exact nature of the groove depth dynamics induced by variable speed of the boundary has not been explored. If the moving boundary achieves a limiting position so its velocity decays, then $d$ will regain its initial value. If the velocity of the grain boundary is always slow, then $d$ will remain constant throughout the development.

In any case, we assume that $d$ is constant throughout the motion, without attempting to spell out the most general conditions under which this is a good assumption.

In this section we provide an analog of the approximation arguments in $\S 3$. Changes are necessary simply because the presence of a nonzero groove angle boundary condition may, by geometry alone, induce a large grain boundary curvature in the direction going into the metal. A large curvature here violates the conditions of applicability of the previous argument.

Our strategy will be to work with an explicit reference grain boundary configuration which conforms to the required boundary angle, but which has no curvature in the direction parallel to the flat surface. When this reference configuration moves parallel to the surface with the appropriate velocity, it is the simplest solution of (2.1) with $u \equiv 1$ which satisfies the boundary conditions. We then look for (not necessarily small) perturbations of this reference configuration, giving us our desired solution of (2.1). We apply, to the perturbation, the same 'Basic assumption' that was proposed originally in $\S 3.1$. This approach generalizes the one in $\S 3$, in which one could think of the reference configuration as being flat and perpendicular to the face. The argument is summarized below in $\S 5.5$. 


\subsection{Assumptions about the groove}

As mentioned before, we assume the depth of the groove is everywhere and always the same, and that the groove angle

$$
0<\theta_{g} \ll 1 \text {. }
$$

The thickness $2 h$ now has the meaning of the distance between the root of the groove on the upper surface and that on the lower surface (Figure 3). The mobile curve $\Gamma_{0}$ is now the locus of the groove root below the upper surface. As before, we first fix attention on a specific point on $\Gamma_{0}$, the origin of our coordinate system, and a specific time $t=0$. We use the same coordinate system $\left(x_{1}, x_{2}, y\right)$ as in $\S 3$.

The boundary condition

$$
\left.\tan ^{-1}\left[\partial_{y} X_{2}\left(x_{1}, 0\right)\right)\right]=-\theta\left(x_{1}, 0\right)=-\theta_{g}
$$

is to be imposed at the upper root, and the same with $+\theta_{g}$ at $y=-2 h$. Because of (5.1), we may approximate (5.2) by

$$
\left.\partial_{y} X_{2}\left(x_{1}, 0\right)\right)=-\theta_{g}
$$

The grain boundary will, as before, span the set $\{-2 h \leqslant y \leqslant 0\}$, but this will no longer be the entire film specimen; just the part between the roots of the two grooves.

The 'basic assumption' given in $\S 3.1$ is no longer necessarily valid, because the boundary condition (5.3) may, when $h$ is small enough, itself force the grain boundary to have a large curvature. When this is the case, the chemical forcing $f(u)$ may not suffice to cause the boundary to move against the force due to curvature. We shall therefore assume this forcing term to be a variable parameter (denoted by $K$ below) and to be large enough for motion to proceed.

The argument in $\S 3.5$ remains valid in the present context, so we shall henceforth assume that $u \equiv 1$. Let $K=f(1)$; as mentioned, we regard it as a variable parameter.

\subsection{Reference profile}

We work with a reference profile

$$
X_{2}^{0}\left(x_{1}, y\right) \equiv X_{2}^{0}(y) \equiv \frac{1}{2} \theta_{g} h\left[-\left(\frac{y}{h}+1\right)^{2}+1\right],
$$

so that $X_{2}^{0}$ satisfies $(5.3), X_{2}^{0}\left(x_{1}, 0\right)=0$, and

$$
\partial_{y}^{2} X_{2}^{0}(y) \equiv \frac{-1}{h} \theta_{g}
$$

Assuming (5.1), we see that the unit normal vector to the surface $\left\{x_{2}=X_{2}^{0}(y)\right\}$ is, up to an error of the order $O\left(\theta_{g}\right)$, directed along the $x_{2}$-axis, i.e. in the plane of the surface of the film. Similarly, the curvature of the reference surface is

$$
H^{0}=-\frac{1}{h} \theta_{g}\left(1+O\left(\theta_{g}\right)\right)
$$

since in (3.2), derivatives of $X_{2}^{0}$ with respect of $x_{1}$ vanish and moreover $a_{22}=1$ when 
$\nabla X_{2}=0$. Therefore this surface, translated in the $x_{2}$-direction with velocity $v_{0}$ given by

$$
\rho v_{0}=-\frac{2}{h} \theta_{g}+K
$$

is an approximate solution of (3.4) with $f(u)$ replaced by $K$. This special solution has no curvature in the plane of the film surface.

We now represent our desired solution at $t=0$ near the origin as a perturbation $X_{2}^{1}$ (not necessarily small) of the reference surface:

$$
X_{2}\left(x_{1}, y\right)=X_{2}^{0}(y)+X_{2}^{1}\left(x_{1}, y\right)
$$

Then (3.4) becomes

$$
\rho v=-\frac{2}{h} \theta_{g}-2\left(a_{11} \partial_{x_{1}}^{2}+a_{12} \partial_{x_{1}} \partial_{y}+a_{22} \partial_{y}^{2}\right) X_{2}^{1}\left(x_{1}, y\right)+K,
$$

which by (5.7)

$$
=\rho v^{0}-2\left(a_{11} \partial_{x_{1}}^{2}+a_{12} \partial_{x_{1}} \partial_{y}+a_{22} \partial_{y}^{2}\right) X_{2}^{1}\left(x_{1}, y\right) .
$$

Let $v=v^{0}+v^{1}$. From (5.10) we have

$$
\rho v^{1}=-2\left(a_{11} \partial_{x_{1}}^{2}+a_{12} \partial_{x_{1}} \partial_{y}+a_{22} \partial_{y}^{2}\right) X_{2}^{1} .
$$

Formally, this is a simple motion-by-curvature problem without forcing. Recall that the $a_{i j}$ are functions of $\nabla X_{2}$, without the superscript "1".

\subsection{Basic assumption eliminating transients when grooving is present}

All terms appearing in (5.11) and (2.2), together with their first spatial derivatives, are bounded independently of $h$.

With this assumption, again designed to eliminate transients, we may proceed as in $\S 3.2$ to deduce that (3.5)-(3.7) hold with $X_{2}$ replaced by $X_{2}^{1}$.

The following argument is the analog of that in $\S 3.3$.

We decompose the curvature by setting $H=H^{0}+H^{1}$, where by neglecting the error terms in (5.6), we have $H^{0}=-\frac{1}{h} \theta_{g}$. Recall $(\S 3.3)$ that the curvature of $\Gamma_{0}$ is denoted by $H_{0}$, and note that

$$
H_{0}=H_{0}^{1},
$$

the latter being $a_{11} \partial_{x_{1}}^{2} X_{2}^{1}$ (see the first term on the right of (5.11)) evaluated at $\nabla X_{2}^{1}=0$, $y=0$. Analogous to (3.8), we obtain

$$
H^{1}-H_{0}^{1} \leqslant C h
$$

Thus

$$
H-\left(H^{0}+H_{0}^{1}\right)=H^{0}+H^{1}-H^{0}-H_{0}^{1}=H^{1}-H_{0}^{1} \leqslant C h .
$$

Note that $H^{0}+H_{0}^{1}$ is independent of $y$.

As in $\S 3.4$, we obtain

$$
v=v_{0}+O\left(h^{2}+\theta_{g}\right),
$$

so that recalling

$$
\rho v=2 H+K,
$$


we get

$$
\rho v_{0}=2\left(H^{0}+H_{0}^{1}\right)+K+O\left(h+\theta_{g}\right)=2 H_{0}^{1}+K_{0}+O\left(h+\theta_{g}\right),
$$

where

$$
K_{0}=K+2 H^{0}=K-\frac{2}{h} \theta_{g} .
$$

Again, we rescale the time variable to effectively make $\rho=1$, use (5.13) and $2 H_{0}=\kappa_{0}$, and neglect the error terms to obtain

$$
v_{0}=\kappa_{0}+K_{0}
$$

This is our new approximate evolution law for $\Gamma_{0}$. It is like (3.23) except that $K_{0}$ replaces 1 .

We assume that

$K_{0}$ is at most of order unity;

then the evolution of $\Gamma_{0}$ is qualitatively like it is for the case of no grooving.

Notice that by (5.18), $K_{0}$ depends upon $h$; therefore in (5.20), we are essentially requiring that the chemical forcing be large enough, for small $h$, to counteract the large second term in (5.18), which is the curvature forced, for small $h$, by the imposed groove angle $\theta_{g}$.

Because of the qualitative similarity, we take note that the computed simulations in $\S 6$ apply to the case when there is grooving, as long as (5.20) holds.

\subsection{Summary}

We rewrote the equation of motion in terms of the deviation $X_{2}^{1}\left(x_{1}, y\right)$ of the grain boundary from an explicit reference configuration incorporating the groove angle, and the deviation $v^{1}$ of the normal velocity from the reference velocity. In terms of the new equation of note (5.11), we then imposed an assumption ( $\$ 5.4)$, analogous to that in $\S 3.1$, and again designed to disregard transients. A final assumption was (5.20) with $K_{0}$ given by (5.18) and $K=f(1)$. On this basis, the law of motion of $\Gamma_{0}$ was found to be (5.19), except for terms negligible when $h$ is small.

\section{Three prototypical cases}

The three cases below, which are simulated numerically, are designed to illustrate three effects associated with bidirectional motion: the formation of double seams, whereby a grain boundary doubles back on itself to encounter a region already in equilibrium $(\S 6.1)$, a threshold effect between evolutions to a stable S-configuration and to a double seam (§6.2), and the migration of a transition point $(\S 6.3)$.

In all cases, we envisage an initial straight grain boundary which is divided into patches which subsequently move in alternating directions. In most cases ( $\$ 6.3$ excluded), enough symmetry is imposed that the mobile grain boundaries are pinned at the transition points between the initial patches.

For purposes of the simulations, the problem is reduced to initial value problems for nonlinear parabolic PDEs. The numerical integration of these equations is by a fourth order Runge-Kutta method in time, with centered differences in space. The coefficients in 
the equations never become singular during the integration, because the quantities $\bar{p}, \hat{p}, p$ in (6.1), (6.2), (6.8) are bounded away from zero. The last term in (6.8) is discontinuous at the creeping transition, but this does not affect the flux. The accuracy of the calculations was checked by doubling the number of grid points.

\subsection{The formation of double seams}

Consider two adjacent patches of the initial grain boundary which begin to migrate in opposite directions. Often it is observed that each of the moving parts of the grain boundary doubles back to impinge on the initial position of the other part (i.e. the other patch). It is not able to proceed immediately into the region which has been covered by the other part, however, but merely advances along that patch without penetrating it. This is illustrated by micrographs and drawings in numerous other papers [2, 5, 7, 8, 11]. The diagrams in these papers sometimes show eventual penetration in some cases; this effect lies outside our theory. What results, then is a configuration in which there are two grain boundaries (really parts of the original grain boundary generated by different patches) moving away from the initial grain boundary's position on either side of it.

Often the initiation of the grain boundary movement does not occur simultaneously in all the patches [5]. The initiation mechanism is no doubt caused by many factors, outside the scope of this theory. Our simulations are for the case of simultaneous initiation.

We have simulated the simplest case in which this occurs, namely the case when the two initial patches are infinite in extent. Then there will be a built-in symmetry which served to keep the transition point between patches fixed

We use the symbols $(x, y)$ for Cartesian coordinates on the upper face; this is a change of notation from before. We formulate a symmetric initial value problem for the motion of $\Gamma_{0}(t)$, which we just write as $\Gamma(t)$, on that face.

The initial shape $\Gamma(0)$ is the $x$-axis. The subsequent velocity is initially upwards for $x<0$, downwards for $x>0$. Thus $\Gamma^{+}(0)=\{x<0\}$ and $\Gamma^{-}(0)=\{x>0\}$.

The prediction is that eventually $\Gamma^{+}$grows to the right and makes contact from above with the positive $x$-axis. But that is an obstacle: the free boundary cannot pass through the positive $x$-axis from above, because it would be entering a region which has already been brought to equilibrium. The equation (4.1) with the last term 1 eliminated would hold there. Since $\kappa<0$ at any point on the positive $x$-axis where $\Gamma$ first arrives, $v$ would have to be $>0$ at that point and time, so entrance is indeed forbidden.

All the above is born out by the numerical simulations in Figures 4 and 5 .

The simulation uses a parabolic partial differential equation representation of (3.23), derived in Appendix B. We represent the interface in the form $\theta=\Theta(r, t),(r, \theta)$ being polar coordinates. Let $w(r, t)=r \Theta(r, t)$. Then

$$
\frac{1}{\bar{p}} w_{t}=\frac{1}{\bar{p}^{3}}\left[w_{r r}+r^{2} \Theta_{r}^{3}\right]-1,
$$

where $\bar{p}^{2}=1+r^{2} \Theta_{r}^{2}$. It is to be solved under the initial condition $w(r, 0)=r \pi$ and boundary conditions $w(0, t)=0, w(r, t) \sim r \pi-t+o(1)$ as $r \rightarrow \infty$.

To handle the obstacle, we drop the last term -1 in (6.1) when $\Theta<0$. The simulations were carried out for the motion of $\Gamma^{+}(t)$; the motion of $\Gamma^{-}(t)$ was then added to the 


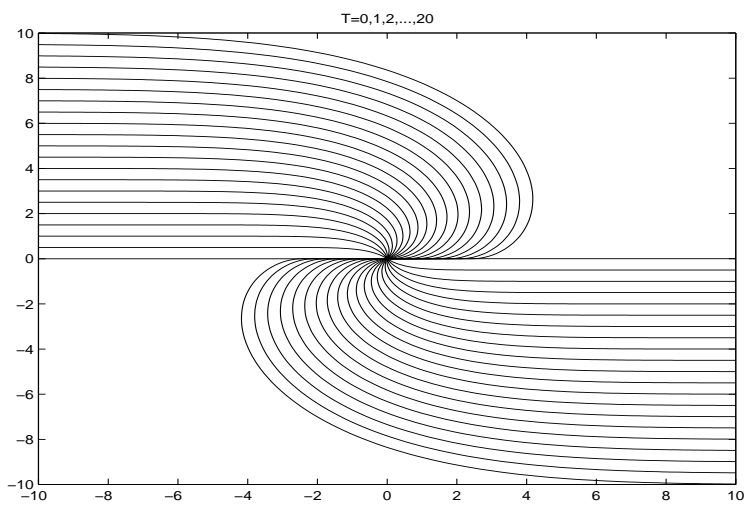

Figure 4. Computer simulation of the formation of a double seam by interfaces doubling back on regions already covered by the grain boundary. The curves depict the positions of the grain boundary at successive times.

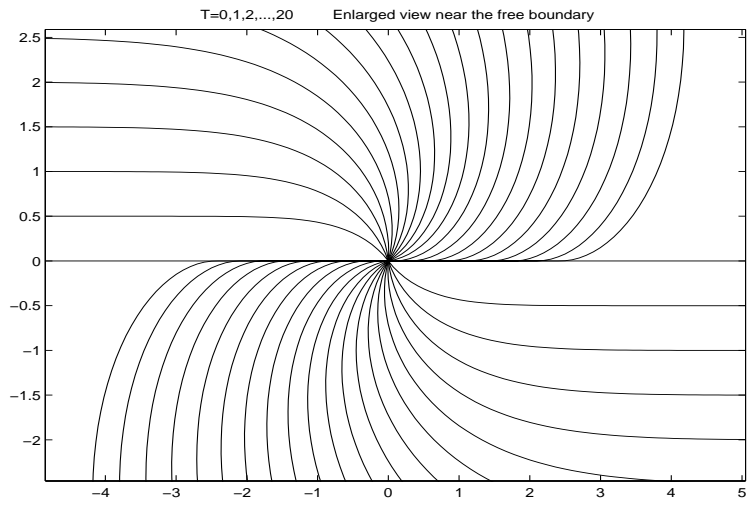

FIGURE 5. Blow-up near the singular point at the origin of the simulations in Figure 4. Each grain portion of the grain boundary flattens against the region already covered by the other.

figure by imposing symmetry. The phenomenon by which one part of the free boundary slides along the opposite initial patch is clearly seen in Figures 4 and 5.

\subsection{Threshold results for a finite $\Gamma$}

Again, we assume enough symmetry that all segments (patches) are pinned at their ends. We envisage the patches being finite, all of them of length $L$. The whole configuration has odd symmetry about $x= \pm L / 2$. The prediction is that if $L \leqslant 2$, the evolution of all patches proceeds to the arc of a circle of radius 1 , because such a circle is an exact stationary solution of (4.1). This final state, with such arcs alternating upward and downward, will be called an S-configuration. But if $L>2$, we may argue that the evolving curve will never attain such a large curvature as 1 . Then the free boundary pieces grow indefinitely, each eventually forming a double seam as in $\S 6.1$. This threshold phenomenon may be justified rigorously; see $\S 6.2 .1$ below. The computation in the subthreshold case is shown in Figure 6, and that in the superthreshold case in Figure 8. The critical case, when the limiting shape is a semicircle, is shown in Figure 7. The initial configuration in these three 


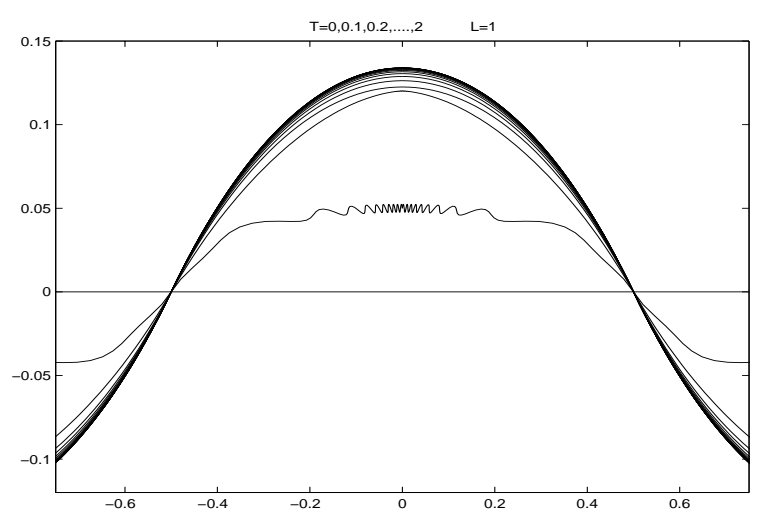

Figure 6. Computed evolution from a pair of adjacent patches, alternating in direction. Here the length of the central patch is subthreshold (length 1) and the evolution approaches a limiting configuration which is a stationary arc of a circle of radius 1 . Note the distortion due to unequal horizontal and vertical scalings. The wiggly line is the imposed configuration at $t=0$. The evolution quickly smooths out the wiggles.

simulations is taken to be irregular with random wiggles. The reason is to show that the wiggles are quickly damped out by the evolution, leaving a solution at later times which appears to be quite independent of the initial data.

Such a threshold phenomenon depending on the size of the patch is not explicitly seen in available micrographs; most of the cited authors appear to believe that S-mechanisms always lead to double seams. The model and analysis given here suggest that reversals of direction of motion may act to inhibit the formation of double seams, and therefore the progress of DIGM. Such a conclusion may have practical implications relating to chemical deterioration of alloys.

For this description, we use the representation $r=R(\theta, t)$, where now $(r, \theta)$ are polar coordinates with origin at $x=-\frac{L}{2}, y=0$. The PDE is now

$$
R_{t}=\hat{p}^{-2}\left[R_{\theta \theta}-\frac{2 R_{\theta}^{2}}{R}-R\right]+\frac{\hat{p}}{R}
$$

$\hat{p}^{2}=R^{2}+R_{\theta}^{2}$. The derivation proceeds on the basis of (6.1) and is given in Appendix B. We use initial and boundary data

$$
R(\theta, 0)=R_{0}(\theta), \quad R(0, t)=L / 2, \quad R_{\theta}\left(\frac{\pi}{2}, t\right)=0 .
$$

Here the initial data $R_{0}(\theta)$ is an arbitrary small positive function describing the initial position of the grain boundary. Any initial irregularities become smoothed out. The graph is then extended to be even with respect to the line $x=0$ and odd with respect to the lines $x= \pm L / 2$.

\subsubsection{Justification of the threshold effect}

The reason for using the representation $r=R(\theta, t)$ and (6.2) was in order to be able to include interfaces such as those in Figure 8 which eventually bulge out and are no longer 


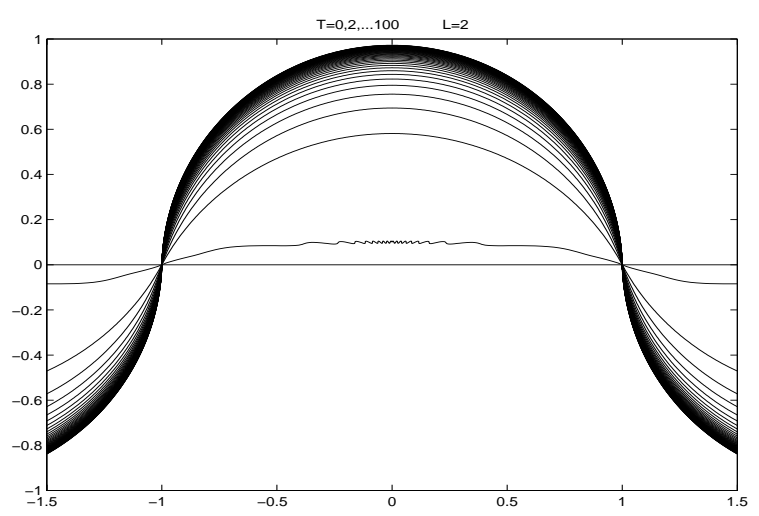

Figure 7. Same as in Figure 6, except that the length of the central patch is 2 rather than 1. The limiting circular arc is exactly a semicircle with radius 1 . This is the critical case.

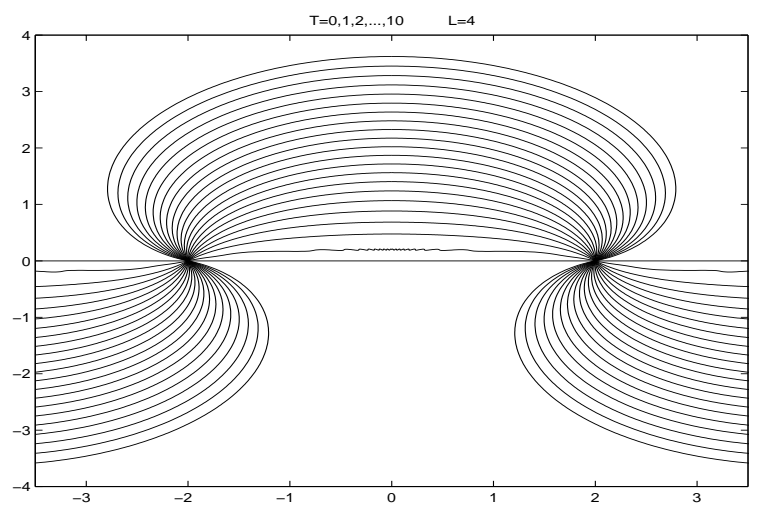

FIGURE 8. Same as in Figure 6, except that the length of the central patch is 4 rather than 1. This is the supercritical case, and the interface increases indefinitely, never approaching a stationary limit configuration.

representable as graphs $y=Y(x, t)$. However, we now move to the easier representation $y=Y(x, t)$, because it will suffice to describe the threshold effect.

We consider only the upward moving patch, with $x$ confined to the basic interval $[-L / 2, L / 2]$. The initial-boundary value problem is then (similar to (6.8) below)

$$
Y_{t}=\frac{1}{p^{2}} Y_{x x}+p
$$

where $p^{2}=1+Y_{x}^{2}$

$$
Y(x, 0)=Y_{0}(x), \quad Y( \pm L / 2, t)=0,
$$

where $Y_{0}(x) \geqslant 0$.

We define a subsolution $\underline{Y}(x, t)$ to be a smooth function satisfying

$$
\underline{Y}_{t} \leqslant \frac{1}{\underline{p}^{2}} \underline{Y}_{x x}+\underline{p}
$$

where $\underline{p}^{2}=1+\underline{Y}_{x}^{2}$. Supersolutions $\bar{Y}$ are defined similarly.

It is a perfectly standard result that if $\underline{Y}, \bar{Y}$ are a sub- and super-solution such that 
$\underline{Y} \leqslant \bar{Y}$ at the two boundary points and also when $t=0$, then this inequality is preserved for later times.

Moreover, if $Y_{0}(x)$ is a stationary subsolution, then the solution $Y(x, t)$ is a nondecreasing function of $t$ for as long as it exists. The (standard) proof of this consists in, first, using the above comparison result to show that $Y(x, t) \geqslant Y_{0}(x)$, and secondly applying that result to the function $v_{\delta}(x, t)=Y(x, t+\delta)-Y(x, t)$ for $\delta>0$. The function $v_{\delta}$ satisfies a similar parabolic PDE and a similar comparison principle. It begins nonnegative and so stays nonnegative. Since $\delta$ is arbitrary, this shows that $Y$ is increasing in $t$.

First, consider the case that $Y_{0}(x) \equiv 0$. It is a subsolution, so the corresponding evolution $Y(x, t)$ must increase in time. We consider two cases:

(1) $L<2$. Then an exact stationary solution of (6.4), $(6.5)_{2}$ exists, namely the function $\tilde{Y}(x) \geqslant 0$ whose graph is the arc of a circle of radius 1 pinned to the boundary points, so that $\tilde{Y}( \pm L / 2)=0$. In fact, it is seen by setting the right-hand side of (3.23) equal to 0 that such arcs are the only stationary solutions. Therefore, by the comparison principle, the function $Y(x, t)$ must be confined below $\tilde{Y}$, exist for all time, and (by the monotonicity in $t$ ) approach it as a limit:

$$
\lim _{t \rightarrow \infty} Y(x, t)=\tilde{Y}(x) \quad \text { for all } x \in[-L / 2, L / 2] .
$$

The existence for all time follows from the a priori bound $Y(x, t) \leqslant \tilde{Y}(x)$, together with $a$ priori estimates for higher derivatives.

This case is illustrated in Figure 6.

(2) Next, suppose $L>2$. Then there exists no such arc of a circle of radius 1 pinned to the boundary points. It follows that $Y(x, t)$ must either increase with $t$ unboundedly or cease to exist at some finite value of $t$.

In fact, the second alternative no doubt is the case, as Figure 8 shows. As a solution of the forced motion-by-curvature equation (3.23), the evolution ceases to satisfy (6.4), (6.5) since it is no longer a graph. Likely $Y_{x}$ will become infinite in finite time at the boundary points $x= \pm L / 2$.

Calculations for the intermediate case $L=2$ are shown in Figure 7. Then the evolution approaches a semicircle, with infinite derivative at the endpoints.

These results were for the special initial condition $Y_{0}(x) \equiv 0$. If the actual initial condition satisfies $0 \leqslant Y_{0}(x) \leqslant \tilde{Y}(x)$, then by the same comparison principle, its graph in Case 1 always lies between $\tilde{Y}(x)$ and the evolution from the special initial condition, which approaches $\tilde{Y}(x)$. Therefore the evolving graph from the arbitrary initial configuration must also approach $\tilde{Y}(x)$, as it does in Figure 6.

Similarly in Case 2, the solution of (6.4), (6.5) no doubt ceases to exist because it lies above an evolution which does.

These considerations were based on a comparison principle for the nonlinear heat equation (6.4), which is not really appropriate for the later stages of the motion. Comparison principles for various generalizations of the motion-by-curvature equation itself abound in the literature; see, for example, Angenent [1]. However, these results apply mainly to curves which do not have pinned ends, as ours do, and so do not appear to be directly applicable to our problem. 


\subsection{A creeping transition point}

Here we investigate the case when the transition point between $\Gamma^{+}$and $\Gamma^{-}$is not pinned, but rather moves in time.

For a computational illustration, we again consider a prototypical situation. We place the origin 0 at the left end of $\Gamma^{+}(0)$, so that $\Gamma^{+}(0)=\left\{0 \leqslant x \leqslant L_{1}\right\}$ and $\Gamma^{-}(0)=\left\{L_{1} \leqslant\right.$ $\left.x \leqslant L_{1}+L_{2}\right\}$. We assume $L_{1}>L_{2}$, so that this much of the previous symmetry is broken. However, we assume odd symmetry with respect to $x=0$ and $x=L_{1}+L_{2}$. This effectively pins the transition points at those locations.

To compute the evolution, we use the representation $y=Y(x, t)$ for both $\Gamma^{ \pm}$, i.e. for $0 \leqslant x \leqslant L_{1}+L_{2}$. The equation is

$$
\frac{1}{p} Y_{t}=\frac{1}{p^{3}} Y_{x x}+h(x, t,[Y]),
$$

where $p^{2}=1+Y_{x}^{2}$, and $h(x, t,[Y])$ depends upon the past history of the function $Y$ as follows:

(a) At points $(x, y, t)$ with $y>0$ which have not been covered by the motion of a grain boundary, $h=1$.

(b) At points $(x, y, t)$ with $y<0$ which have not been covered by the motion of a grain boundary, $h=-1$.

(c) At points $(x, y, t)$ which have been covered by the motion of a grain boundary, $h=0$.

The initial and boundary conditions are

$$
Y(x, 0)=0, \quad Y(0, t)=Y\left(L_{1}+L_{2}, t\right)=0 .
$$

Initially at $t=0$, we set $h=\left\{\begin{array}{l}1, \quad x<L_{1}, \\ -1, \quad x>L_{1}\end{array}\right.$

The prediction is that the transition point will migrate to the right, maybe eventually swallowing up $\Gamma^{-}$. This indeed is shown in the figure.

The movement of the transition point is not clear from the available micrographs; however those in Baumann et al. [2] are suggestive of this.

\section{Discussion}

We have built upon the previous models for DIGM $[3,6,4]$ in a systematic manner to account for effects not covered in them. These effects are associated with the migration of curved bidirectional grain boundaries spanning a thin metallic film.

To obtain this information, we have followed these steps:

(1) We have extended the asymptotic reduction from phase field model to free boundary problem given in Fife et al. [6] so that problems in a film with a 3D geometry can be handled. The reduction is based on the smallness of a parameter measuring the thickness of the grain boundary and the strength of the elastic interaction.

(2) We have further reduced this $3 \mathrm{D}$ FBP to a $2 \mathrm{D}$ one, this time using approximations based on the thinness of the film and assumptions on the solution calculated to ensure that transient effects have passed away. The derived free boundary problem 


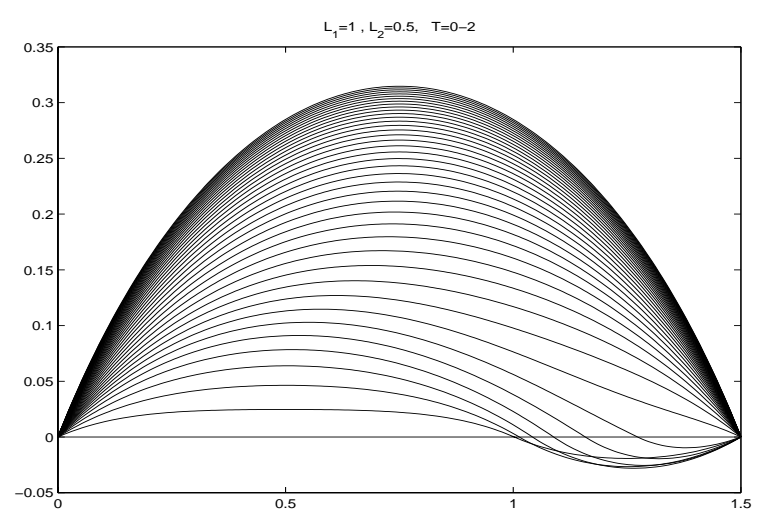

FIGURE 9. In this case, the transition point between the portions of the grain boundary moving in opposite directions is the intersection of the boundary with the $x$-axis. It begins at $x=1$ and is not fixed, but rather migrates in such a way that the smaller of the two adjacent patches becomes even smaller and eventually vanishes. Odd symmetry is imposed at $x=0$ and $x=1.5$, so the picture is periodic with period 1.5. Only one period interval is shown.

in this case is a simple one of forced motion by curvature, the forcing function taking positive, negative, or zero values depending on the situation. In the case when there is a non-neglible grooving angle, the elimination of transient effects becomes more complicated. In order for the grain boundary to move, it must be assumed that the solute concentration is large enough to overcome an inhibiting effect associated with the groove angle. This critical amount can be estimated by means of a reference configuration which we introduce.

(3) The final FBP is solved numerically for several prototypical configurations. Specifically, the well known formation of S-configurations and double seams is illustrated by computer simulations of the free boundary problem. Moreover a threshold effect and the phenomenon of creeping transition point are predicted and illustrated.

Regarding the threshold effect, the model and analysis given here suggest that reversals of direction of motion may act to inhibit the formation of double seams, and therefore the progress of DIGM. Such a conclusion may have practical implications relating to chemical change in alloys.

\section{Acknowledgements}

PCF's research was supported by NSF Grant DMS-9703483, and by The Hong Kong University of Science and Technology during his visit there. We are grateful to J. W. Cahn for bringing to our attention the phenomenon of grooving and for valuable discussions.

\section{Appendix A Derivation of the 3D free boundary problem}

We detail the modifications to the derivation in Fife et al. [6] needed to obtain (2.1), (2.2). To avoid repetition of that long and complex argument, we simply point out the changes that may be made for this purpose in the various steps of the previous derivation. 


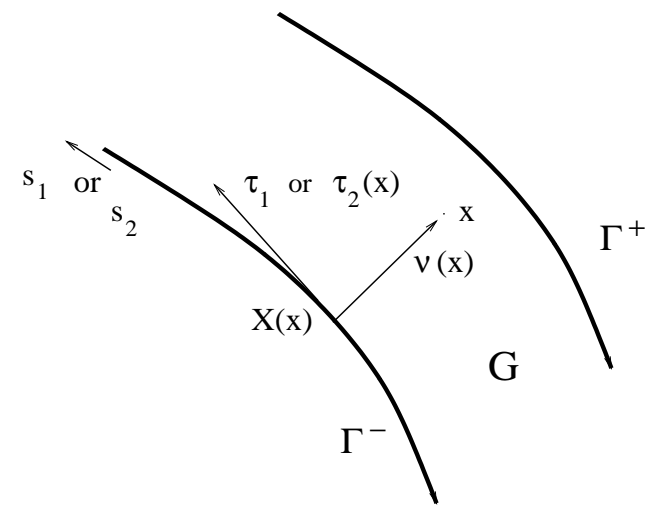

FIGURE A 1. Coordinate system in the grain boundary.

References to equations in [6] will be made by the notation 'I' placed before the equation number from Fife et al. [6].

Our starting point will be the same as in Fife et al. [6], namely the system I(2.10)-I(2.12) of PDE's for the phase function $\phi$, concentration $c$, and chemical potential $w$. The grain boundary $G(t)$, defined by $\mathrm{I}(2.15)$, will now be a mobile curved shell in $3 \mathrm{D}$, rather than a strip in 2D. For the purpose of this section, unidirectional motion will still be assumed; thus $G(t)$ has a leading surface $\Gamma^{+}(t)$ and a trailing surface $\Gamma^{-}(t)$, on which $\mathrm{I}(2.16)$ holds.

First we review some simple geometric concepts. For now we fix $t$, and in fact disregard time dependence. As in Fife et al. [6], for $x \in G$ we define $X(x)$ to be the point on $\Gamma^{-}$ closest to $x, r(x)=|x-X(x)|=$ distance from $x$ to $\Gamma^{-}$, and $\boldsymbol{v}(x)$ the unit vector in the direction from $X(x)$ to $x$ (since $G$ is open, $r(x)>0$ and $\boldsymbol{v}(x)$ is well defined). For any function $f(x)$, we represent

$$
\nabla f(x)=\boldsymbol{v}(x) \partial_{r} f(x)+\tau_{\mathbf{1}}(x) \partial_{1} f(x)+\tau_{2}(x) \partial_{2} f(x),
$$

where $\tau_{i}(x)$ are chosen in any smooth fashion so that $\left(v, \tau_{1}, \tau_{2}\right)$ form an orthonormal triple. Here $\partial_{r} f$ is the derivative in the direction of $\boldsymbol{v}$, and the tangential derivatives $\partial_{i} f$, $i=1,2$, are in the directions of $\tau_{i}$. Thus for any function $w(x)$,

$$
\begin{gathered}
\nabla \cdot D \nabla w=\left(v \partial_{r}+\sum_{1}^{2} \tau_{i} \partial_{i}\right) \cdot D\left(v \partial_{r} w+\sum_{1}^{2} \tau_{i} \partial_{i} w\right) \\
=\partial_{r}\left(D \partial_{r} w\right)+\sum_{1}^{2} \tau_{i} \cdot \partial_{i}\left(D v \partial_{r} w\right)+\sum_{1}^{2} v \cdot \partial_{r}\left(\tau_{i} D \partial_{i} w\right)+R w,
\end{gathered}
$$

where the second order differential operator $R$ involves only $\partial_{1}$ and $\partial_{2}$, not $\partial_{r}$. Note that as we move from $X(x)$ to $x$ along the normal to $\Gamma^{-}, \boldsymbol{v}(x)$ is unchanged, but a twisting may cause $\tau_{i}$ to change.

We now fix a point $x_{0}$ and let $s_{1}, s_{2}$ denote arclengths along two curves (which we think of as coordinate curves) on $\Gamma^{-}$with origin at $X\left(x_{0}\right)$, tangent at that point to $\tau_{1}\left(x_{0}\right)$ and $\tau_{2}\left(x_{0}\right)$, respectively (Figure A 1 ). We also require the curves to lie in planes normal to $\Gamma^{-}$ at the origin. Then the curves will be the intersections of $\Gamma^{-}$with the planes through $X\left(x_{0}\right)$ determined by $\boldsymbol{v}\left(x_{0}\right)$ and either $\tau_{1}\left(x_{0}\right)$ or $\tau_{2}\left(x_{0}\right)$. Let the curvatures of the two curves 
at 0 be denoted by $\kappa_{i}\left(x_{0}\right)$, deemed positive if the respective centre of curvature lies on the same side of $\Gamma^{-}$as $G$. Then $\left(r, s_{1}, s_{2}\right)$ form a local coordinate system in a neighborhood of $x_{0}$, so that for a function $f(x)$, we may write $f=\tilde{f}\left(r, s_{1}, s_{2} ; x_{0}\right)$ and express, by the dilatation effect as in I(D.1), the operator $\partial_{i}$ appearing in (A 2) as

$$
\partial_{i} f\left(x_{0}\right)=\left.\frac{1}{1-r\left(x_{0}\right) \kappa_{i}\left(x_{0}\right)} \partial_{s_{i}} \tilde{f}\left(r, s_{1}, s_{2} ; x_{0}\right)\right|_{s_{1}=s_{2}=0} .
$$

Attaching a similar meaning to $\tilde{\boldsymbol{v}}\left(r, s_{1}, s_{2} ; x_{0}\right)$, we also know that $\left.\partial_{s_{i}} \tilde{\boldsymbol{v}}\right|_{s_{1}=s_{2}=0}=$ $-\kappa_{i}\left(x_{0}\right) \tau_{i}\left(x_{0}\right)$. Although $\partial_{r} \tau_{i}(x)$ does not generally vanish (due to twisting), it will be orthogonal to $v(x)$, so that $v(x) \cdot \partial_{r} \tau_{i}(x)=0$. Therefore the second summation on the right of (A 2) vanishes. In (A 2) we may write

$$
\partial_{i}\left(D \boldsymbol{v} \partial_{r} w\right)=\boldsymbol{v} \partial_{i}\left(D \partial_{r} w\right)+\frac{D \partial_{r} w}{1-r \kappa_{i}} \partial_{s_{i}} \tilde{\boldsymbol{v}}
$$

so that since $v \cdot \tau_{i}=0$,

$$
\tau_{i} \cdot \partial_{i}\left(D v \partial_{r} w\right)=-\kappa_{i} \frac{D \partial_{r} w}{1-r \kappa_{i}} .
$$

Thus (A 2) becomes the following for any point $x$, no longer fixed:

$$
\nabla \cdot D \nabla w=\partial_{r}\left(D \partial_{r} w\right)-\left(\sum_{1}^{2} \frac{D \kappa_{i}}{1-r \kappa_{i}}\right) \partial_{r} w+R w
$$

where $R$ is a second order differential operator of the form $R w=\sum_{(i, j)} b_{i}^{1}(x) \partial_{i}\left[D b_{j}^{2}(x) \partial_{j} w\right]$ for some $b_{i}^{1}, b_{j}^{2}$. This equation (A 6) replaces I(D.6) except for the factor $\rho^{-1}$, and the right side of $\mathrm{I}(2.25)$ becomes (A 6) with $r$ replaced by $\epsilon z$ and $w$ by $W$. As in Fife et al. [6], it is now appropriate to change the metric on $\Gamma^{-}$by $\mathrm{I}(2.17)$. This introduces a factor $\rho^{-1}$ in front of $R W$ in (A 6).

We now consider the other parts of $\mathrm{I}(2.24), \mathrm{I}(2.25)$. The right side of $\mathrm{I}(2.24)$ becomes, by setting $D=1$ in the above,

$$
\Phi+\Phi_{z z}-\sum_{1}^{2} \frac{\epsilon \kappa_{i}}{1-\epsilon z \kappa_{i}} \Phi_{z}-\epsilon p_{\phi}+\epsilon^{2} \hat{R} \Phi
$$

where $\hat{R} \Phi$ involves derivatives of $\Phi$ in directions other than $r$.

Consider the left side of I(2.24). We now restore $t$-dependence, and give a different meaning to the coordinates $s_{i}$. That curvilinear coordinate system will now be fixed, rather than attached to the variable point $X(x)$. Let $\left(x_{1}, x_{2}, y\right)$ be Cartesian coordinates; we specify that the film occupy the set $\{-2 h<y<0\}$ as in Figure 2 of the present paper and Section 2.5 of Fife et al. [6] (replace $x_{2}$ there by $y$ ). Thus $\left(x_{1}, x_{2}\right)$ are Cartesian coordinates for the top and bottom faces of the film. Let $\Gamma_{0}(t)=\Gamma(t) \cap\{y=0\}$ (this is the top curve in Figure 2), and $s_{0}$ be arclength along $\Gamma_{0}(t)$ from some reference point, as was done with the coordinate $s$ in Fife et al. [6] (\$2.2).

Let $P\left(t, s_{0}\right)$ be the plane perpendicular to $\Gamma_{0}(t)$ at $s_{0}$, and $\Gamma_{1}\left(t, s_{0}\right)=\Gamma(t) \cap P\left(t, s_{0}\right)$. Consider points $x \in G(t)$ such that $X(x) \in \Gamma_{1}\left(t, s_{0}\right)$. For given $x$, let $s_{0}(x, t)$ be the value of $s_{0}$ for which this is true.

Let $s_{1}(x, t)$ be the arclength along $\Gamma_{1}\left(t, s_{0}\right)$ from $\Gamma_{0}(t)$ to the closest point $X(x, t)$ to $x$. In particular, each point on $\Gamma^{-}(t)$ is specified by a pair $\left(s_{0}, s_{1}\right)$. 
To summarize, the coordinate transformation $(x, t) \rightarrow\left(r(x, t), s_{0}(x, t), s_{1}(x, t)\right)$ is defined as follows. We identify $X(x, t)$ as the closest point on $\Gamma^{-}$. This also defines the plane $P=P\left(t, s_{0}\right)$ through $X$ normal to $\Gamma_{0}(t)$, hence $s_{1}(x, t)$ and $s_{0}(x, t)$, the latter being the arclength from the reference point on $\Gamma_{0}$ to $P$.

In the coordinate system $\left(z, s_{0}, s_{1}\right)(z=r / \epsilon$ as in Fife et al. [6] $)$, time derivatives at $t=0$ are transformed as follows, analogous to I(2.19):

$$
\phi_{t}(x, 0)=\Phi_{t}\left(z, s_{0}, s_{1}, 0\right)+\epsilon^{-1} r_{t}(x, 0) \Phi_{z}\left(z, s_{0}, s_{1}, 0\right)+\sum_{i=1}^{2} \partial_{t} s_{i}(x, 0) \partial_{s_{i}} \Phi\left(z, s_{0}, s_{1}, 0\right) .
$$

Let $v\left(s_{0}, s_{1}, t\right)$ be the normal velocity of $\Gamma^{-}(t)$. Also let $v_{0}\left(s_{0}, t\right)$ be the normal velocity of $\Gamma_{0}(t), \kappa_{0}\left(s_{0}, t\right)$ its curvature, and let $v_{0}^{*}$ be given by $\mathrm{I}(2.30)$ with $s_{i}^{*}$ defined by $\mathrm{I}(2.17)$. As in Fife et al. [6], we derive $\partial_{t} s_{0}(x, t)=\rho^{-1} S_{1}\left(s_{0}, t\right)$, where $S_{1}\left(s_{0}, t\right)=\int_{0}^{s_{0}} v_{0}^{*}\left(\rho^{-1 / 2} s^{\prime}, t\right) \kappa_{0}\left(s^{\prime}, t\right) d s^{\prime}$. Also $\partial_{t} s_{1}$ is given by such an analogous expression $\rho^{-1} S_{2}$, whose exact form is not important.

In all, we have the following equations to replace $\mathrm{I}(2.24)$ and $\mathrm{I}(2.25)$. In them, the symbol $s$ will now mean $\left(s_{0}, s_{1}\right)$, and similarly for $s^{*}$.

$$
\begin{gathered}
\rho \epsilon^{2} \Phi_{t}(z, s, t)-\rho \epsilon v(s, t) \Phi_{z}(z, s, t)+\epsilon^{2}\left(S_{1}\left(s^{*}, t\right) \Phi_{s_{0}}(z, s, t)+S_{2}\left(s^{*}, t\right) \Phi_{s_{1}}(z, s, t)\right) \\
=\Phi+\Phi_{z z}-\epsilon \sum_{1}^{2}\left(\frac{\kappa_{i}}{1-\epsilon z \kappa_{i}}\right) \Phi_{z}-\epsilon p_{\phi}+\epsilon^{2} \hat{R} \Phi \\
\epsilon U_{t}\left(z, s^{*}, t\right)-v(s, t) U_{z}\left(z, s^{*}, t\right)+\epsilon \rho^{-3 / 2}\left(S_{1}\left(s^{*}, t\right) U_{s_{1}^{*}}(z, s, t)+S_{2}\left(s^{*}, t\right) U_{s_{2}^{*}}(z, s, t)\right) \\
=\epsilon^{-2}\left(D W_{z}\right)_{z}-\epsilon^{-1} \sum_{1}^{2}\left(\frac{D \kappa_{i}}{1-\epsilon z \kappa_{i}}\right) W_{z}+\rho^{-1} R W .
\end{gathered}
$$

Finally, I(2.26) still holds.

The assumptions listed at the beginning of $\S 2.3$ of [6] still hold, with minor modifications. In Assumption I, $\kappa_{1}, \kappa_{2}$ both have the regularity stated. The integral condition now is applied only to $\kappa_{0}$, as a function of $s_{0}$, since the analogous condition resulting in the boundedness of $S_{2}$ is automatically fulfilled by virtue of the bounded length of $\Gamma_{1}$. Assumption IV still holds with the obvious changes of meaning for the derivatives.

$\S 2.3 .1$ is still valid, with the evident new interpretation of $\mathrm{I}(2.47), \mathrm{I}(2.50)$. Also, only obvious changes are needed in Section 2.3.2.

Changes in $\S 2.4$ result, as we shall show, in the new FBP $(2.1),(2.2)$ to replace $\mathrm{I}(2.74)$, $\mathrm{I}(2.75)$.

To see this, we first consider $M(t)(\mathrm{I}(2.61))$. We now consider two pairs of numbers $s_{01}^{*}<s_{02}^{*}, \quad s_{11}^{*}<s_{12}^{*}$ and define $G^{*}(t)=G(t) \cap\left\{\rho^{1 / 2} s_{01}^{*}<s_{0}<\rho^{1 / 2} s_{02}^{*}, \quad \rho^{1 / 2} s_{11}^{*}<s_{1}<\right.$ $\left.\rho^{1 / 2} s_{12}^{*}\right\}$.

The increase in dimension means that the boundary $\partial G^{*}$ now contains four lateral portions rather than 2 . We call the union of the lateral portions $\partial_{L} G^{*}$.

Obvious changes in the treatment of $F_{1}$ result in the same estimate $\mathrm{I}(2.64)$.

Consider now $F_{2}$. The brackets in $\mathrm{I}(2.65)$ and $\mathrm{I}(2.67)$ are to be replaced by an integral over $\partial_{L} G^{*}$. In fact, the first equation in $\mathrm{I}(2.67)$ becomes $F_{2}=\int_{\partial_{L} G^{*}} \rho^{-1 / 2} \tau \cdot D \nabla w$. However, for each $z=z_{0}$, by the divergence theorem, $\int_{\partial_{L} G^{*} \cap\left\{z=z_{0}\right\}} \tau \cdot D \nabla W=\int_{G^{*} \cap\left\{z=z_{0}\right\}} \nabla \cdot D \nabla W$. 
Therefore

$$
F_{2}=\int_{0}^{\pi} \rho^{-1 / 2} d z^{\prime} \int_{G^{*} \cap\left\{z=z^{\prime}\right\}} \nabla \cdot D \nabla W(1+O(\epsilon)),
$$

and by the same chain of estimates as in [6], we find the analog of I(2.68):

$$
F_{2}=\pi D_{0} \rho^{-1 / 2} \int_{G^{*} \cap\{z=\pi / 2\}} \Delta^{*} U+\rho^{-1 / 2} O\left(\hat{\epsilon}+\epsilon \rho^{-1 / 2}\right),
$$

where $\Delta^{*}$ is the Laplacian on the surface $G^{*} \cap\{z=\pi / 2\}$ with respect to the starred metric.

Continuing the argument as in Fife et al. [6], we obtain (2.1) and (2.2). (In Fife et al. [6] and Cahn \& Penrose [4], $f(u)$ was taken to be $u^{2}$ in accord with the theory in Cahn et al. [3], but here it could be any nonnegative increasing function.)

\section{Appendix B The equation of motion in polar coordinates}

\section{B.1 Derivation of the equation for $w$}

We consider the scenario described in $\S 6.1$. Let $(r, \theta)$ be polar coordinates, so that $x=r \cos \theta$, etc. We represent the moving curve $\Gamma(t)$ in the form $\theta=\Theta(r, t)$, and show that for $x<0$, (4.1) reduces to (6.1).

Let $\tau(r, t), \boldsymbol{v}(r, t)$ be the tangential and normal unit vectors; also $\boldsymbol{e}_{r}(\theta), \boldsymbol{e}_{\theta}(\theta)$ are the unit vectors in the directions of the ray from the origin in the direction $\theta$, and in the perpendicular direction with $\theta$ increasing.

We first represent $\tau$ and $\boldsymbol{v}$ in terms of $\boldsymbol{e}_{r}$ and $\boldsymbol{e}_{\phi}$. We have

$$
\boldsymbol{d} \boldsymbol{x}=\boldsymbol{e}_{r} d r+\boldsymbol{e}_{\phi} d \theta .
$$

For points restricted to lie on $\Gamma$ with fixed $t$, we have $d \theta=\Theta_{r} d r$, so

$$
\boldsymbol{d} \boldsymbol{x}=\left(\boldsymbol{e}_{r}+r \Theta_{r} \boldsymbol{e}_{\phi}\right) d r .
$$

If $s$ is arclength on $\Gamma, \tau=\frac{d \boldsymbol{x}}{d s}=\left(\boldsymbol{e}_{r}+r \Theta_{r} \boldsymbol{e}_{\phi}\right) \frac{d r}{d s}$, so that $\frac{d r}{d s}=\frac{1}{\sqrt{1+r^{2} \Theta_{r}^{2}}}$ and

$$
\tau=\frac{\boldsymbol{e}_{r}+r \Theta_{r} e_{\phi}}{\sqrt{1+r^{2} \Theta_{r}^{2}}}, \quad v=\frac{r \Theta_{r} e_{r}-e_{\phi}}{\sqrt{1+r^{2} \Theta_{r}^{2}}} .
$$

For the curvature, we have

$$
-\kappa \boldsymbol{v}=\frac{d \tau}{d s}=\frac{1}{\sqrt{1+r^{2} \Theta_{r}^{2}}} \frac{d}{d r} \frac{\boldsymbol{e}_{r}+r \Theta_{r} \boldsymbol{e}_{\phi}}{\sqrt{1+r^{2} \Theta_{r}^{2}}} .
$$

We calculate

Hence

$$
\frac{d}{d \theta} \boldsymbol{e}_{r}=\boldsymbol{e}_{\phi}, \quad \frac{d}{d \theta} \boldsymbol{e}_{\phi}=-\boldsymbol{e}_{r}
$$

$$
\frac{d}{d r}\left(\boldsymbol{e}_{r}+r \Theta_{r} \boldsymbol{e}_{\phi}\right)=\Theta_{r} \frac{d}{d \theta} \boldsymbol{e}_{r}+\frac{d}{d r}\left(r \Theta_{r}\right) \boldsymbol{e}_{\phi}+r \Theta_{r}^{2} \frac{d}{d \theta} \boldsymbol{e}_{\phi}=\left(r \Theta_{r r}+e \Theta_{r}\right) \boldsymbol{e}_{\phi}-r \Theta_{r}^{2} \boldsymbol{e}_{r}
$$

Also $\frac{d}{d r}\left(r^{2} \Theta_{r}^{2}\right)=2 r \Theta_{r}^{2}+2 r^{2} \Theta_{r} \Theta_{r r}$. Thus letting

$$
\bar{p}=\sqrt{1+r^{2} \Theta_{r}^{2}},
$$


we have

$$
\frac{d}{d r} \frac{\boldsymbol{e}_{r}+r \Theta_{r} \boldsymbol{e}_{\phi}}{\sqrt{1+r^{2} \Theta_{r}^{2}}}=\frac{1}{\bar{p}}\left[\left(r \Theta_{r r}+2 \Theta_{r}\right) \boldsymbol{e}_{\phi}-r \Theta_{r}^{2} \boldsymbol{e}_{r}\right]-\frac{1}{2} \bar{p}^{-3}\left(2 r \Theta_{r}^{2}+2 r^{2} \Theta_{r} \Theta_{r r}\right)\left(\boldsymbol{e}_{r}+r \Theta_{r} \boldsymbol{e}_{\phi}\right),
$$

and from (B 3), (B 4),

$$
-\kappa \boldsymbol{v}=\bar{p}^{-2}\left[\left(r \Theta_{r r}+2 \Theta_{r}\right) \boldsymbol{e}_{\phi}-r \Theta_{r}^{2} \boldsymbol{e}_{r}\right]-\bar{p}^{-4}\left(r^{2} \Theta_{r} \Theta_{r r}+r \Theta_{r}^{2}\right)\left(\boldsymbol{e}_{r}+r \Theta_{r} \boldsymbol{e}_{\phi}\right) .
$$

Use (B 3) together with $\kappa=-(\kappa v) \cdot v$ to get

$$
\begin{gathered}
\kappa=-\frac{r \Theta_{r}}{\bar{p}}\left[-r \Theta_{r}^{2} \bar{p}^{-2}-\bar{p}^{-4}\left(r^{2} \Theta_{r} \Theta_{r r}+r \Theta_{r}^{2}\right)\right] \\
+\frac{1}{\bar{p}}\left[\bar{p}^{-2}\left(r \Theta_{r r}+2 \Theta_{r}\right)-\bar{p}^{-4} r \Theta_{r}\left(r^{2} \Theta_{r} \Theta_{r r}+r \Theta_{r}^{2}\right)\right] \\
=\bar{p}^{-3} r^{2} \Theta_{r}^{3}+\bar{p}^{-5} r^{2} \Theta_{r}^{2}\left(r \Theta_{r r}+\Theta_{r}\right)+\bar{p}^{-3}\left(r \Theta_{r r}+2 \Theta_{r}\right)-\bar{p}^{-5} r^{2} \Theta_{r}^{2}\left(r \Theta_{r r}+\Theta_{r}\right) \\
=\bar{p}^{-3}\left(r \Theta_{r r}+2 \Theta_{r}+r^{2} \Theta_{r}^{3}\right) .
\end{gathered}
$$

We now represent the velocity $v$. Since $\boldsymbol{x}=\boldsymbol{r e}_{r}(\Theta(r, t))$, we have

$$
\boldsymbol{d} \boldsymbol{x}=\left(\boldsymbol{e}_{r}+r \Theta_{r} \frac{d}{d \theta} \boldsymbol{e}_{r}\right) d r+r \Theta_{t} \frac{d}{d \theta} \boldsymbol{e}_{r} d t=\left(\boldsymbol{e}_{r}+r \Theta_{r} \boldsymbol{e}_{\phi}\right) d r+r \Theta_{t} \boldsymbol{e}_{\phi} d t .
$$

To find the normal velocity, we choose $d r$ so that $\boldsymbol{d x}$ is in the direction of $\boldsymbol{v}$, i.e. $\boldsymbol{d} \boldsymbol{x} \cdot \boldsymbol{\tau}=0$. From (B 3), we need $\boldsymbol{d} \boldsymbol{x} \cdot\left(\boldsymbol{e}_{r}+r \Theta_{r} \boldsymbol{e}_{\phi}\right)=0$ :

$$
\begin{gathered}
d r+\left(r \Theta_{r} d r+d \Theta_{t} d t\right) r \Theta_{r}=0, \\
d r=-\bar{p}^{-2} r^{2} \Theta_{r} \Theta_{t} d t .
\end{gathered}
$$

Put this into (B 10) to obtain

$$
\boldsymbol{d} \boldsymbol{x}=\frac{1}{\bar{p}^{2}}\left[-r^{2} \Theta_{r} \boldsymbol{e}_{r}+r \boldsymbol{e}_{\phi}\right] \Theta_{t} d t=-\frac{v r \Theta_{t}}{\bar{p}} d t
$$

Thus

$$
v=-\frac{r \Theta_{t}}{\bar{p}}
$$

is the normal velocity.

From (B 13), (B 9), we may write the law of motion (B 2) as

$$
\frac{r \Theta_{t}}{\bar{p}}=\frac{1}{\bar{p}^{3}}\left(r \Theta_{r r}+2 \Theta_{r}+r^{2} \Theta_{r}^{3}\right)-1 .
$$

Because of the factor $r$ in two terms of (B 14), the problem is singular at $r=0$, and we cannot prescribe a priori $\theta(0, t)$. We wouldn't in fact expect to. This can be somewhat alleviated by definiting $w(r, t)=r \Theta(r, t)$, so $w_{r r}=r \Theta_{r r}+2 \Theta_{r}, w_{r}(0, t)=\Theta(0, t)$. Then (B 14) becomes

$$
\frac{1}{\bar{p}} w_{t}=\frac{1}{\bar{p}^{3}}\left(w_{r r}+r^{2} \Theta_{r}^{3}\right)-1,
$$

which is (6.1). We solve it under the boundary and initial conditions given following (6.1). 


\section{B.2 Derivation of the equation for $R$}

For fixed $t$, the function $r=R(\theta, t)$ is the inverse function to $\theta=\Theta(r, t)$. Therefore in (B 14) we may make the replacements

$$
\Theta_{r}=1 / R_{\theta}, \quad \Theta_{t}=-R_{t} / R_{\theta}, \quad \Theta_{r r}=-R_{\theta \theta} /\left(R_{\theta}\right)^{3} .
$$

Doing this results in (6.2).

\section{References}

[1] Angenent, S. (1991) Parabolic equations for curves on surfaces Part II. Intersection, blow-up and generalized solutions. Annals of Math. 133, 171-215.

[2] Baumann, S. F., Michael, J. \& Williams, D. B. (1981) Initiation and growth of the grain boundary discontinuous precipitation reaction. Acta Metall. 29, 1343-1355.

[3] Cahn, J. W., Fife, P. C. \& Penrose, O. (1997) A phase-field model for diffusion-induced grain boundary motion. Acta Mater. 45, 4397-4413.

[4] Cahn, J. W. \& Penrose, O. (2001) Theory of curvature and grooving effects in DIGM. In preparation.

[5] Duty, D. \& BRechet, Y. (1994) Nucleation mechanism of discontinuous precipitation in Mg-Al alloys and relation with the morphology. Acta Metall. Mater. 42, 3035-3043.

[6] Fife, P. C., Cahn, J. W. \& Elliott, C. M. (2001) A free boundary model for diffusion induced grain boundary motion. Interfaces and Free Boundaries, 3, 291-336.

[7] Fournelle, R. A. (1979) Discontinuous coarsening of lamellar cellular precipitate in an austenitic Fe-30 wt.\%Ni-6 wt.\%Ti alloy-I. Morphology. Acta Metall. 27, 1135-1145.

[8] Frebel, M. \& Schenk, J. (1979) On the growth of the discontinuous precipitation reaction at individual grain boundaries in $\mathrm{Au}-40$ at. \% Fe alloys. Z. Metallkde. 70, 230-240.

[9] Muluins, W. W. (1957) Theory of thermal grooving. J. Applied Phys. 28, 333-339.

[10] Mullins, W. W. (1958) The effect of thermal grooving on grain boundary motion. Acta Metall. 6, 414-417.

[11] Williams, D. B. \& ButLeR, E. P. (1981) Grain boundary discontinuous precipitation reactions. Int. Metals Reviews, 3, 153-183. 